\title{
Performance Analysis of the Effect of Nonlinear Low Noise Amplifier for Wideband Spectrum Sensing in the Poisson Field of Interferers
}

\author{
Bipun Man Pati and Attaphongse Taparugssanagorn \\ Telecommunications, Asian Institute of Technology, P.O. Box 4, Klong Luang, Pathumthani 12120, Thailand
}

Correspondence should be addressed to Bipun Man Pati; bemaanpati@gmail.com

Received 11 March 2016; Revised 9 October 2016; Accepted 17 October 2016

Academic Editor: Rui Zhang

Copyright (C) 2016 B. Man Pati and A. Taparugssanagorn. This is an open access article distributed under the Creative Commons Attribution License, which permits unrestricted use, distribution, and reproduction in any medium, provided the original work is properly cited.

A cognitive radio (CR) device likely consists of a low-cost low noise amplifier (LNA) due to the mass-production reason. Nevertheless, the operation of a low-cost LNA becomes highly nonlinear causing intermodulation (IM) interference. The most important task of CR devices is to sense the wideband spectrum to increase opportunistic throughput. In noncooperative secondary networks, the IM interference usually can be ignored for the narrowband spectrum sensing, while the IM interference needs to be taken into account along with interference from other CR devices in the wideband case. Our contribution is to study the effects of a nonlinear LNA for the second case in environments modeled by Poisson field of interferers reflecting more realistic scenario. As shown in the simulation results, the performance of the receiver is degraded in all the cases due to the nonlinearity of LNA. The adaptive threshold setting based on the multivariate Gaussian mixture model is proposed to improve the receiver performance.

\section{Introduction}

The number of wireless devices is growing constantly. One common reason is the increasing mobile penetration; that is, the costs of access and devices, for example, smart phones and tablets, are coming down. No matter what the device is, it utilizes radio frequency (RF) spectrum as its channels for its communications. As a result, RF spectrum plays a very important role in wireless communications. The current $\mathrm{RF}$ allocation, which is used by the federal communications commission (FCC), defines specific RF bands for specific uses. For example, there are RF bands allocated for cellular communications, military communications, marine, amplitude modulation (AM)/frequency modulation (FM), and so forth. This method of frequency allocation results in heavy utilization of some RF bands and very poor utilization of some other RF bands [1]. Hence, the efficient utilization of RF spectrum is a major challenge in today's wireless communication systems.
CR is a software defined radio (SDR) that has the ability to sense the environment and adjust its RF parameters to provide opportunistic access to secondary user (SU) on the frequency band of primary user (PU). In the context of CR, PUs or licensed users are those who have legacy right for the frequency band and SUs are those who opportunistically access the frequency band of PU. Since CRs are secondary users, one of the requirements of $\mathrm{CR}$ is to reliably detect the existence of $\mathrm{PU}$ in a given frequency band before actually utilizing that band for communication purpose. The other requirement is that when utilizing the frequency band of $\mathrm{PU}$, it is very important for CR that they do not interfere with the PU. This requirement leads to the spectrum sensing as the first and most crucial task of CR.

The CR front-end can support either narrowband or wideband spectrum sensing. In narrowband spectrum sensing, a very narrowband of spectrum is sensed to decide if what is sensed is really signal with noise or just noise. If the band consists of signal with noise the secondary user 
is prohibited to use that band and is allowed if the band consists of only noise. While in the present CR performs narrowband spectrum sensing, in the long run, the $C R$ needs to perform sensing over wide range of frequency increasing the overall opportunistic throughput [2]. Wideband spectrum sensing senses spectral opportunities in a wide range of frequency. Determining spectral holes in wideband spectrum can be done using channel by channel scanning approach [3]. But this method of detecting spectrum holes requires an RF front-end with many tunable narrow band-pass filters. This results in higher implementation complexity. As a result, a direct conversion receiver (DCR) has become very common as receiver for wideband spectrum sensing. Although superheterodyne receiver is also an alternative solution, several advantages of DCR, for instance, the circumvention of the image problem, no use of image filter, and surface acoustic wave (SAW) filter [4], over the superheterodyne technique make it suitable for CRs. Therefore, the DCR is generally preferred over the superheterodyne receivers.

A received signal in wideband spectrum sensing has high dynamic range; as a result, the receiver operates at the nonlinear region. This causes nonlinear distortion and adversely affects the sensing decision of the spectrum sensing algorithms. The components that give rise to the nonlinearity property in the DCR are an RF filter, an LNA, and a mixer. The nonlinear distortion is caused by IM and cross-modulation (XM) terms that occur when the signal passes through the nonlinear components of the receiver. These terms might fall within the region of the desired signal in case of the wideband spectrum sensing. It is noted that these IM distortion (IMD) terms cannot be filtered out as in the narrowband sensing case since these terms overlap with the PU signal being sensed. Among other components in the DCR, the most significant source of nonlinearity is LNA [5].

With regard to interference modeling in a CR application, the interference from a CR network to a PU network has received a lot more attention in the literature $[6,7]$. This is obvious since the CR users must not interfere with the PU. The study of interference from PUs to a CR network has been reported in a number of papers $[8,9]$. In [9], they study the interference to a CR network due to several PUs and derive outage probability distribution for the entire CR network. It does not consider the effect of the intranetwork interference on a single CR sensing node. The performance of only a single spectrum sensing node due to the intranetwork interference in noncooperative environment was studied in [10]. However, this paper assumed that all the interfering signals are colocated spectrally and did not take into account the effect of nonlinearity presented in the CR. The effect of receiver nonidealities has been studied in a number of papers [11-13]. These studies consider narrowband spectrum sensing and assume that the receiver operates in the linear region. References $[14,15]$ consider wideband spectrum sensing and study the effect of third-order nonlinearity due to LNA in the performance degradation of the energy detector and the cyclostationary detector. These papers study only the effect of IMD terms in the frequency band. However, in noncooperative $\mathrm{CR}$ network considering wideband spectrum sensing the frequency band contains interfering signal from other CRs in addition to IMD terms.

In [10] authors studied the effect of intranetwork interference in noncooperative environment assuming that the interfering signals are colocated spectrally which is good but it does not cover the situation where the interfering signal may be located on separate frequency bands. Furthermore, it does not consider the effect of strong in-band interfering signal on the sensing node due to the nonlinearity of LNA in DCR. A more realistic study would be to consider the situation where CRs may be transmitting either on either separate frequency bands or same frequency band considering the effect of strong in-band interfering signal. References $[14,15]$ investigated effect of IMD on the performance of sensing node but they do not consider the scenario where the subband of interest may be degraded further by other interfering signals in a noncooperative environment which would be more realistic; for example, the subband at which the sensing node is detecting the PU signal is degraded not only by IMD but also by the interfering signal from other CRs in a noncooperative network.

Our contribution is to study the performance of detector in wideband spectrum sensing in noncooperative environment considering the effect not only of IMD but also due to the interfering signal from other CRs which has not been investigated before in $[10,14,15]$. We investigate the performance of energy detector for wideband spectrum sensing considering the effect of nonlinearity in LNA. In particular, we focus on a noncooperative environment. We consider a more realistic scenario in a noncooperative network where the interfering signals are generated based on the Poisson point process. The Poisson model enables the statistical characterization of network interferer taking into account the spatial distribution as well as the density of the interfering nodes. We model the interference in a wideband spectrum sensing at a particular subband not only by the IMD terms but also due to the interfering signals from other CRs. The model presented provides more realistic viewpoint for studying the performance of detector in a noncooperative network that has not yet been studied in the literature. We identify two more realistic scenarios in such noncooperative network and derive the expression for false alarm and detection probability making use of the central limit theorem (CLT) for each of the two scenarios. By relaxing our model in the distribution of interference and IMD terms we model a very realistic scenario present in a noncooperative network. Finally, we proposed an adaptive threshold setting method that is based on modeling the noise after the nonlinear LNA using multivariate Gaussian mixture model. We investigated by modeling the noise signal after LNA; using the multivariate Gaussian mixture model the performance of detector is enhanced.

The rest of the paper is organized as follows. In Section 2, we describe the system model for noncooperative network along with the interferer and LNA nonlinearity model. In Section 3, we first derive the expression for the detection and the false alarm probabilities considering various cases and then provide the simulation results for those cases. We also discuss the adaptive threshold setting based on multivariate 


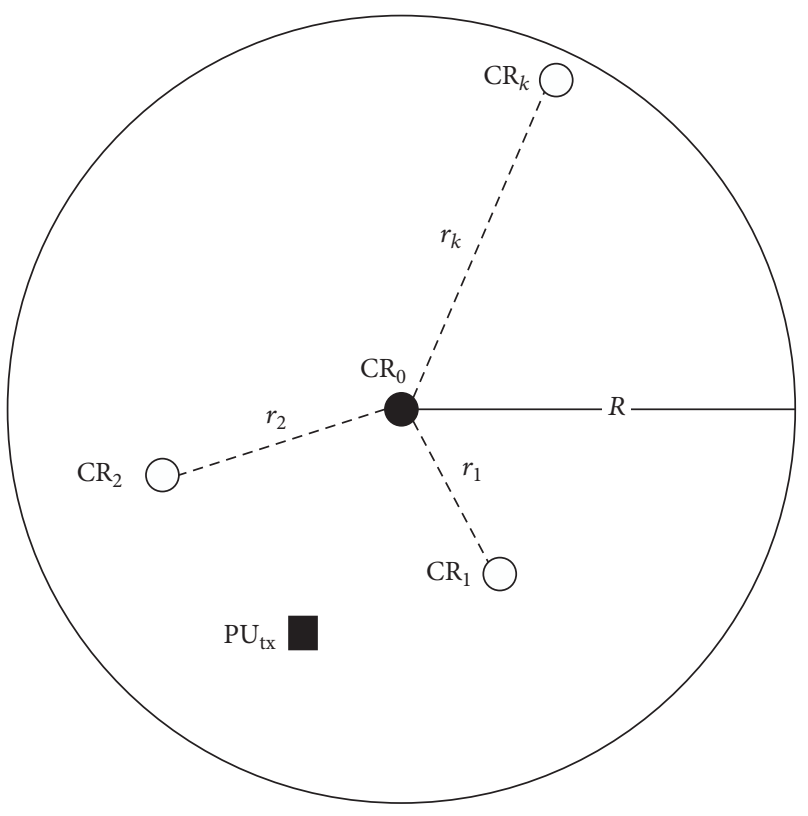

FIGURE 1: System model.
Gaussian mixture model for improving the performance in case of LNA nonlinearity. Finally, conclusions are drawn in Section 4 .

\section{System Model}

The system under consideration is shown in Figure 1. We consider a circular geographical area with the radius $R$ containing $K$ number of CRs, a central $\mathrm{CR}\left(\mathrm{CR}_{0}\right)$, and a $\mathrm{PU}$. The distance between $\mathrm{CR}_{0}$ and $K$ th $\mathrm{CR}\left(\mathrm{CR}_{K}\right)$ is denoted by $r_{K}$. The distance between $\mathrm{CR}_{0}$ and $\mathrm{PU}$ can be varied as required. The $\mathrm{PU}$ is the licensed user and has higher priority for transmission than CRs. However, the network policy allows CRs to use the spectrum with the condition that they must not cause harmful interference to the PU.

2.1. Interferer Modeling. The spatial distribution of network interferers is modeled as a Poisson point process in a twodimensional circular plane. The spatial density of interfering nodes is denoted by $\lambda_{\mathrm{IN}}$ in the number of CRs per unit area. The probability that $k$ number of nodes interfere at any particular time follows the Poisson distribution as expressed in (1). The interfering nodes are defined as the set of terminals which transmit within the frequency band of interest during the sensing time and hence effectively contribute to the total interference.

$$
\operatorname{Pr}(K=k)=\frac{(\mu(A))^{k} e^{-\mu(A)}}{k !}
$$

where $\mu(A)=\lambda_{\mathrm{IN}} \pi R^{2}$.
The distribution of a CR around the circular area is considered to be uniform. Hence, the distribution of $r$ is given by

$$
f_{R}(r)= \begin{cases}\frac{2 r}{R^{2}}, & \text { for } 0<r<R \\ 0, & \text { otherwise. }\end{cases}
$$

2.2. Modeling Nonlinearity due to LNA. During the sensing time interval of $\mathrm{CR}_{0}$, the other CRs transmit data in various frequency bands. This is plausible because the CR network is noncooperative; that is, one CR may not know the status and location of the other CRs. If $\mathrm{CR}_{0}$ is close to some other CRs, then strong interference in-band signal is dominated by $\mathrm{CR}_{0}$. This strong in-band signal forces the LNA to operate in the nonlinear region. As a result, the spurious frequency components, such as IM and XM, occur with some other bands that are possibly in the band of interest. The even-order nonlinearity terms are outside the frequency range while the odd-order nonlinearity terms remain within the frequency range. The complex baseband equivalence of the wideband signal after the nonlinear LNA is written as follows [18]:

$$
y[n]=f(x[n])+w_{a}[n],
$$

where $x[n]$ is the received complex baseband equivalent wideband signal and $w_{a}[n]$ is the additive white Gaussian noise (AWGN) after the LNA.

The complex baseband equivalent wideband signal in (3) is written as

$$
x[n]=s[n]+\sum_{k=1}^{K}\left(c_{k}[n]\right)+w_{b}[n],
$$

where $s[n]$ is the PU signal, $c_{k}[n]$ is the signal from $k$ th CR, and $w_{b}[n]$ is the AWGN before LNA.

Since the third-order nonlinearity often dominates over the higher order nonlinearity components, a memoryless polynomial model up to the third-order is used to model the effect of nonlinearity due to the LNA. The third-order memoryless polynomial model consists of the nonlinear coefficients up to the third-order. Thus, $f(x[n])$ in (3) can be written as

$$
f(x[n])=\beta_{1}(x[n])+\beta_{2}(x[n])^{2}+\beta_{3}(x[n])^{3},
$$

where $\beta_{1}, \beta_{2}$, and $\beta_{3}$ are the coefficients of the memoryless polynomial model.

Considering only odd-order nonlinearity in (5) and using (3), the final expression for the signal is given by

$$
y[n]=\beta_{1}(x[n])+\beta_{3}(x[n])^{3}+w_{a}[n] .
$$

The values for $\beta_{1}, \beta_{2}$, and $\beta_{3}$ are related to the circuit specification parameters. $\beta_{1}$ is the small signal gain and its typical value is $35 \mathrm{~dB}$, the value of $\beta_{2}$ can be calculated using the second-order intercept point (IP2) coefficient, and the value of $\beta_{3}$ can be calculated using (7) [16]. 


$$
\begin{aligned}
& A_{\mathrm{IP} 3}=\sqrt{\frac{4 \beta_{1}}{3 \beta_{3}}}, \\
& P_{\mathrm{IP} 3}=20 \log _{10} A_{\mathrm{IP} 3}+10 \mathrm{dBm},
\end{aligned}
$$

where $A_{\mathrm{IP} 3}$ and $P_{\mathrm{IP} 3}$ are the amplitude and power at thirdorder intercept point (IP3) of the LNA.

\section{Spectrum Sensing Method}

A cyclostationary detection (CD) performs spectrum sensing by correlating the received signal with its frequency shifted version. The frequency by which the signal is to be shifted is called a cyclic frequency. The cyclic frequency is a function of cyclic feature, for instance, the signal modulation type, its symbol rate, and the carrier frequency. Depending upon the presence or the absence of the cyclic feature, the decision is made to determine the presence or absence of PU signals. A CD takes advantage of the fact that noise is a wide sense stationary (WSS) process with no correlation, whereas the modulated signals are cyclostationary with their spectral correlation and their Fourier transform, the cyclic spectral density (CSD) [8]. The CSD of the received signal is computed as

$$
\sum_{\tau=-\infty}^{\infty} R_{y}^{\alpha}(\tau) \exp (-j 2 \pi f \tau),
$$

where the cyclic autocorrelation function $(\mathrm{CAF}) R_{y}^{\alpha}(\tau)$ is given by

$$
R_{y}^{\alpha}(\tau)=E\left[y(n+\tau) y^{*}(n-\tau) \exp (-j 2 \pi \alpha n)\right],
$$

where $\alpha$ is the cyclic frequency which is assumed to be known or it can be extracted and be used for identifying the transmitted signal.

An energy detection (ED) is the special case of the CD with the lag and the cyclic frequency both equal to zero. Therefore, the test statistics under the ED is given by

$$
R_{y}^{0}(0)=\frac{1}{N} \sum_{n=0}^{N-1}|y[n]|^{2} .
$$

Let $\lambda_{\mathrm{ED}}$ be the threshold to be compared; then the probability of false alarm $P_{\mathrm{fa}}$ and the probability of detection $P_{d}$ under those two mentioned cases are given by

$$
\begin{gathered}
P_{\mathrm{fa}}=\operatorname{Pr}\left\{R_{y}^{0}(0)>\lambda_{\mathrm{ED}} \mid H_{0}\right\}, \\
P_{d}=\operatorname{Pr}\left\{R_{y}^{0}(0)>\lambda_{\mathrm{ED}} \mid H_{1}\right\} .
\end{gathered}
$$

3.1. $P_{d}$ and $P_{f a}$ without LNA Nonlinearity and Interference. When the observation interval $N$ is sufficiently large, the test statistics for ED can be approximated as a Gaussian distribution [19] due to the central limit theorem (CLT). The test statistics can then be approximated by

$$
f_{R \mid H_{0}}(r) \sim \mathcal{N}\left(\sigma_{n}^{2}, \frac{2 \sigma_{n}^{4}}{N}\right),
$$

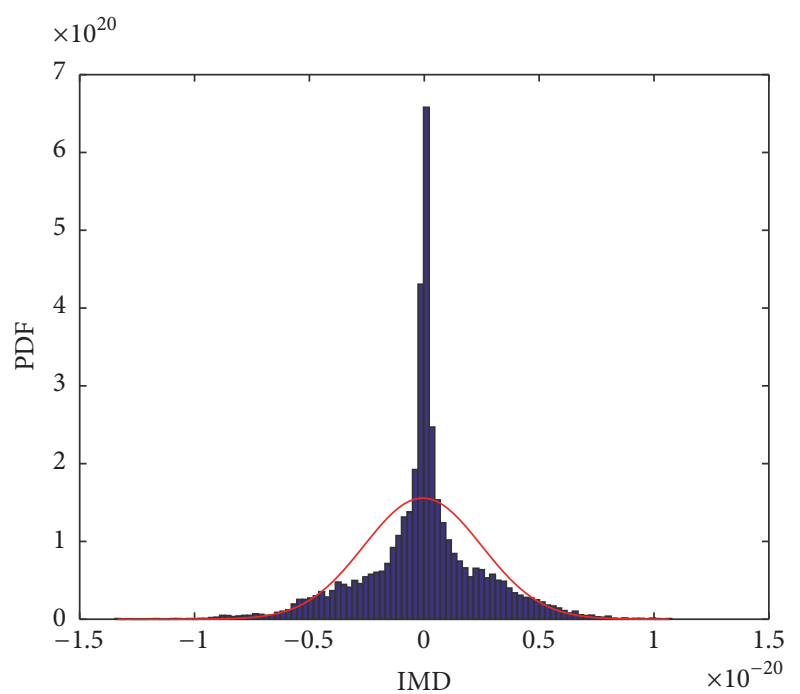

FIgURE 2: Third-order IMD term distribution.

where $\sigma_{n}^{2}$ is the noise variance and $\mathcal{N}(a, b)$ is a Gaussian distribution with mean $a$ and variance $b$.

$$
f_{R \mid H_{1}}(r) \sim \mathcal{N}\left(\sigma_{y}^{2}, \frac{2 \sigma_{y}^{4}}{N}\right),
$$

where $\sigma_{y}^{2}=\sigma_{s}^{2}+\sigma_{n}^{2}$ is the total variance of signal plus noise and $\sigma_{s}^{2}$ is the signal variance.

Using (12) and (14) we derive $P_{d}$ as (15) and using (11) and (13) we derive $P_{\mathrm{fa}}$ as (16):

$$
P_{d}=Q\left(\sqrt{\frac{N}{2}}\left(\frac{\lambda}{\left(\sigma_{n}^{2}+\sigma_{s}^{2}\right)}-1\right)\right),
$$

where the signal to noise ratio (SNR) is defined as $\sigma_{s}^{2} / \sigma_{n}^{2}$, and

$$
P_{\mathrm{fa}}=Q\left(\frac{\lambda-\sigma_{n}^{2}}{\sigma_{n}^{2} / \sqrt{N / 2}}\right),
$$

where $Q(\cdot)$ is the $Q$-function. Under $H_{0}$ hypothesis, for a fixed $P_{\mathrm{fa}}$, the threshold is derived as

$$
\lambda_{\mathrm{ED}}=\sigma_{n}^{2}\left(1+\frac{\mathrm{Q}^{-1}\left(P_{\mathrm{fa}}\right)}{\sqrt{N / 2}}\right),
$$

where $Q^{-1}(\cdot)$ is the inverse $Q$-function. $\lambda_{\mathrm{ED}}$ in (17) indicates that the threshold estimation is dependent on the noise variance, the signal variance, the number of the samples, and the required false alarm probability.

3.2. Distribution of IMD Terms. The IMD is common and critical in the wideband spectrum sensing. The distribution of IMD term plays a vital role in the detection performance of any detector. The fitting of the IMD distribution with the stable distribution yields the following parameters: $\alpha$ close to $1, \beta$ close to 0 , and $\gamma$ and $\delta$ equal to 0 . For simplicity, we approximate this distribution as a Gaussian distribution. The effect of IMD on the performance of detector is shown through several graphs in Section 3. The distribution of IMD term is shown in Figure 2. 
TABLE 1: Simulation parameters for non-Poisson field of interferer.

\begin{tabular}{lc}
\hline Parameters & Values \\
\hline SNR & $-15: 30 \mathrm{~dB}$ \\
SBR (signal to blocker ratio) & $-24:-30 \mathrm{~dB}$ \\
IMD & $2: 10 \mathrm{~dB}$ \\
$\beta_{1}$ & $56.23[16]$ \\
$\beta_{3}$ & $-7497.33[16]$ \\
$\rho$ (noise power uncertainty) & $0.001 \mathrm{~dB}[17]$ \\
\hline
\end{tabular}

3.3. $P_{d}$ and $P_{f a}$ with LNA Nonlinearity. It is assumed that the signal, the IMD term, and the noise are all mutually statistically independent and tend to a Gaussian distribution for large $N$. Using the CLT we approximate the test statistics under two hypotheses as

$$
f_{R \mid H_{0}}(r) \sim \mathcal{N}\left(\sigma_{n}^{2}+\sigma_{\text {imd }}^{2}, \frac{2\left(\sigma_{n}^{2}+\sigma_{\text {imd }}^{2}\right)^{2}}{N}\right),
$$

where $\sigma_{n}^{2}$ is the noise variance and $\sigma_{\text {imd }}^{2}$ is the IMD term variance.

$$
f_{R \mid H_{1}}(r) \sim \mathcal{N}\left(\sigma_{y}^{2}, \frac{2 \sigma_{y}^{4}}{N}\right)
$$

where $\sigma_{y}^{2}=\sigma_{s}^{2}+\sigma_{n}^{2}+\sigma_{\text {imd }}^{2}$ is the total variance with the signal, the noise, and the IMD term.

Using (12) and (19) we derive $P_{d}$ as (20) and using (11) and (18) we derive $P_{\mathrm{fa}}$ as (21):

$$
P_{d}=\mathrm{Q}\left(\sqrt{\frac{\bar{N}}{2}}\left(\frac{\lambda_{\mathrm{ED}}}{\left(\sigma_{n}^{2}+\sigma_{s}^{2}+\sigma_{\mathrm{imd}}^{2}\right)}-1\right)\right)
$$

where the signal to IMD ratio (SIMDR) is defined as $\sigma_{s}^{2} / \sigma_{\text {imd }}^{2}$, and

$$
P_{\mathrm{fa}}=Q\left(\frac{\lambda_{\mathrm{ED}}-\left(\sigma_{n}^{2}+\sigma_{\mathrm{imd}}^{2}\right)}{\left(\sigma_{n}^{2}+\sigma_{\mathrm{imd}}^{2}\right) / \sqrt{N / 2}}\right)
$$

Under hypothesis $H_{0}$, for a fixed $P_{\mathrm{fa}}$, the threshold is derived as

$$
\lambda_{\mathrm{ED}}=\left(\sigma_{n}^{2}+\sigma_{\mathrm{imd}}^{2}\right)\left(1+\frac{Q^{-1}\left(P_{\mathrm{fa}}\right)}{\sqrt{N / 2}}\right) .
$$

$\lambda_{\mathrm{ED}}$ in (22) indicates that the threshold estimation is dependent on the noise variance, the SNR, the number of the samples, the required false alarm probability, and the IMD variance.

3.4. Simulation Results with and without LNA Nonlinearity. Table 1 lists the system parameters used in the simulations for the case of the nonlinearity in LNA.

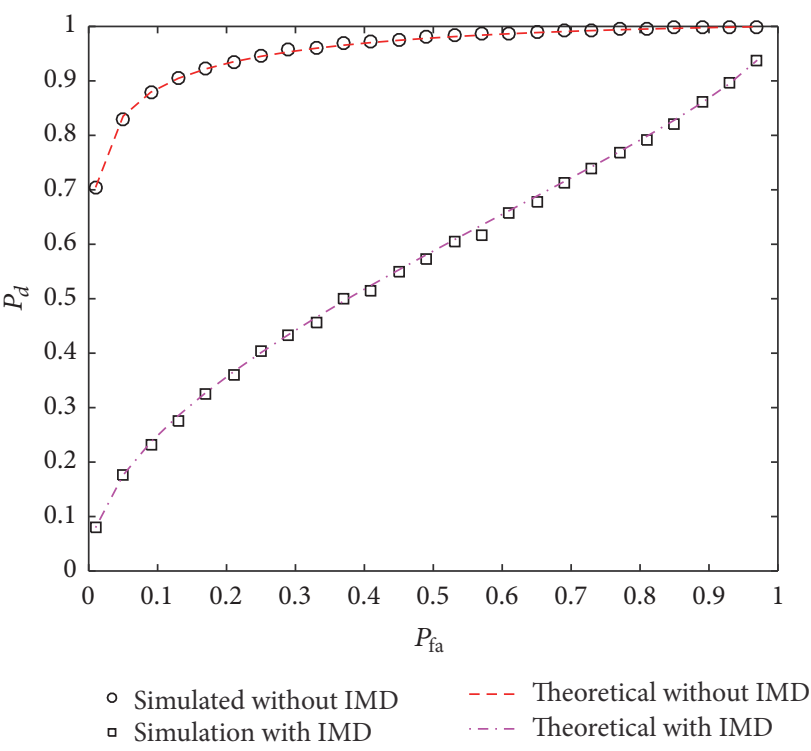

FIgure 3: $P_{\mathrm{fa}}$ versus $P_{d}$ with SNR at $-15 \mathrm{~dB}$, IMD of $10 \mathrm{~dB}$, and $N=$ 1000.

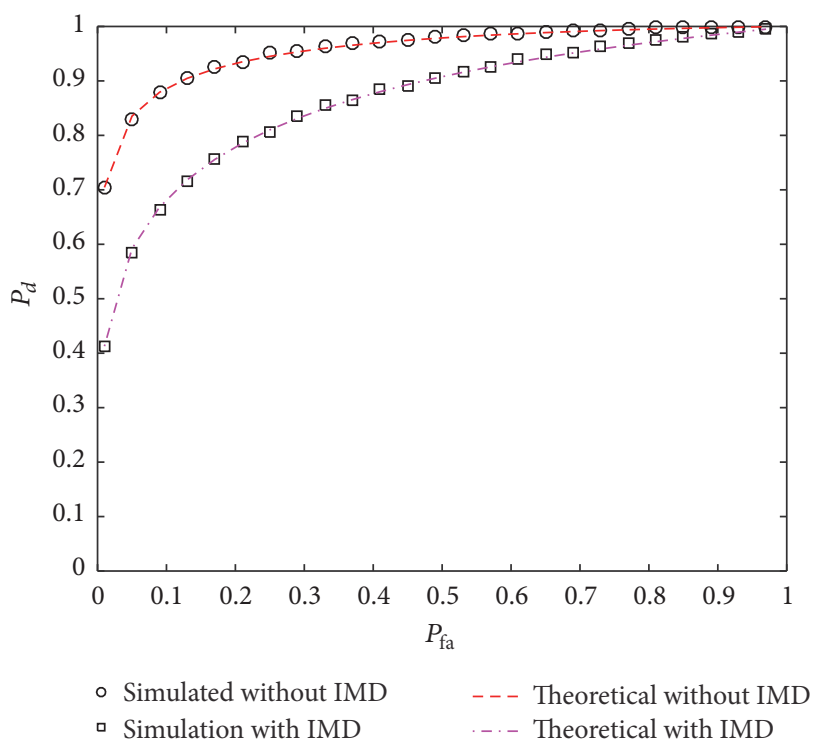

FIgUre 4: $P_{\mathrm{fa}}$ versus $P_{d}$ with SNR at $-15 \mathrm{~dB}$, IMD of $2 \mathrm{~dB}$, and $N=$ 1000 .

Receiver Operating Characteristic Curve for ED. The receiver operating characteristic (ROC) curve is obtained by plotting $P_{\mathrm{fa}}$ along the $x$-axis versus $P_{d}$ along the $y$-axis. We vary the value of $P_{\mathrm{fa}}$ from 0.01 to 1 with an increment of 0.01 at each step. Keeping SNR at $-15 \mathrm{~dB}$ and IMD at $10 \mathrm{~dB}$ and the observation interval at $N=1000$, the ROC curve obtained is shown in Figure 3. If we reduce the power of IMD term to $2 \mathrm{~dB}$, we obtain the ROC curve as shown in Figure 4; the improvement in ROC curve is seen. 


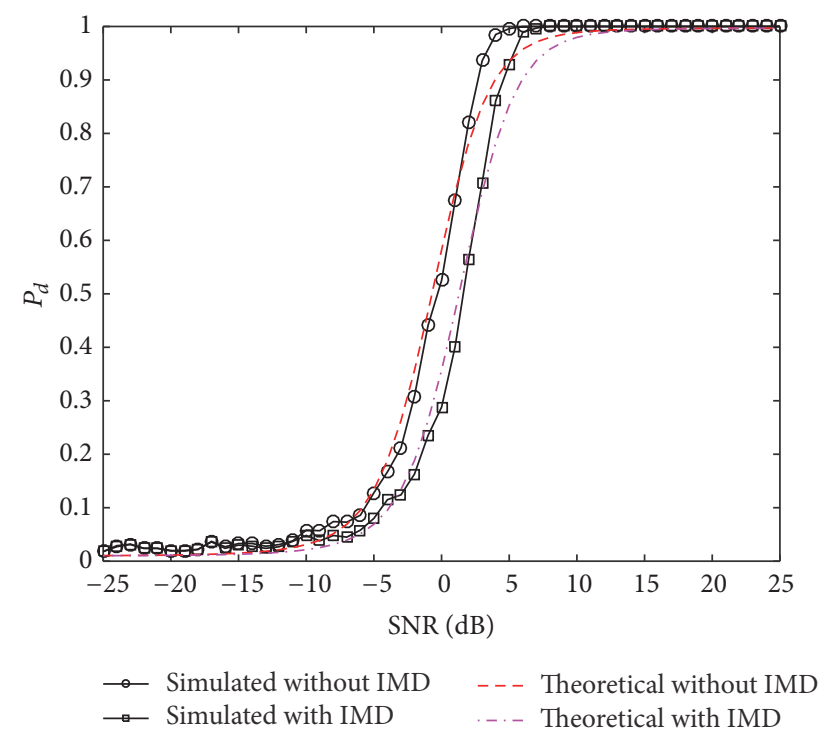

Figure 5: $P_{d}$ versus SNR with IMD of $2 \mathrm{~dB}$ and $N=15$.

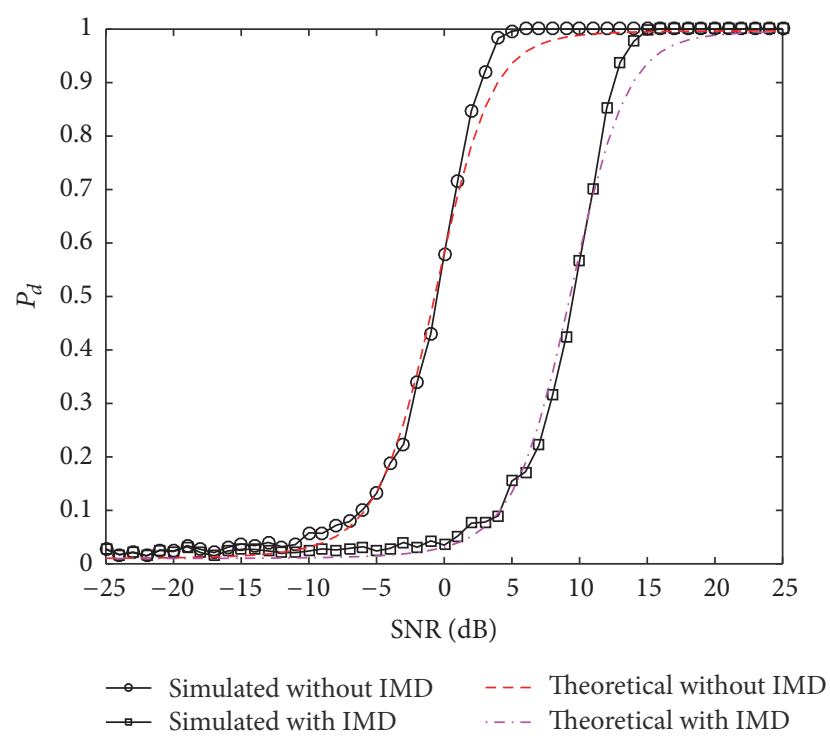

FIGURE 6: $P_{d}$ versus SNR with IMD of $10 \mathrm{~dB}$ and $N=15$.
3.5. Spectrum Sensing under Nonlinear LNA considering Interference. The received signal from each of the interfering nodes is given by (23). Interfering signals are considered independent of each other. The power of $k$ th interferer at a distance of $r_{k}$ with respect to the central CR $\left(\mathrm{CR}_{0}\right)$ is $P_{k}=r_{k}^{-\gamma}$, where $\gamma$ is the path loss exponent. The complex baseband equivalence wideband signal received by the $\mathrm{CR}_{0}$ from other CRs is given by

$$
c[n]=\sum_{k=1}^{K} \sqrt{P_{k}} c_{k}[n]
$$

where $c_{k}[n]$ is the baseband signal from $\mathrm{CR}_{K}$.

As shown in system model, the $\mathrm{CR}_{0}$ is sensing the wideband of spectrum; we suppose that there are in total $M$ subchannels that are sensed by the central CR. The received signal by the central CR depends on whether the network is cooperative or noncooperative. In this paper we discuss noncooperative network. Under cooperative network, there is no effect of interference or nonlinearity, since when a CR is sensing spectrum, the other CRs are considered to be quite. This scenario can be modeled as the case of spectrum sensing previously discussed. As mentioned earlier, in a noncooperative network, CRs have no idea about the location and the status of the other CRs. Hence, one CR interferes with the other CR resulting in the degradation of spectrum sensing. For the narrowband spectrum sensing, the effect of nonlinearity is not relevant but the effect of interferer from the other CRs is relevant. However, in the case of the wideband spectrum sensing, the effect of nonlinearity due to the LNA should also be considered in addition to interference from the other CRs. Under the noncooperative case the following two scenarios are possible:

(i) All the CRs transmit on separate frequency bands.

(ii) A number of CRs transmit on the same frequency band.

CR Transmission on Separate Frequency Bands. When all the CRs transmit on separate frequency band, the received complex baseband equivalent wideband signal before the LNA under two hypotheses is given by

$$
x[n]= \begin{cases}w_{b}[n]+\sum_{k=1}^{K} c_{k}[n], & H_{0}, \\ w_{b}[n]+s[n]+\sum_{k=1}^{K} c_{k}[n], & H_{1} .\end{cases}
$$
performance of the detector is $P_{d}$ versus SNR. The plot of $P_{d}$ versus SNR at IMD of $2 \mathrm{~dB}$ at $P_{\mathrm{fa}}=0.01$ and observation interval $N=15$ is shown in Figure 5 . Now, to analyze the effect of IMD, we plot the same graph keeping all the parameters constant and increase IMD to $10 \mathrm{~dB}$; the plot obtained is shown in Figure 6. Comparing to the graphs plotted in Figures 5 and 6, it is concluded that when the IMD is low, the required $P_{d}$ can be achieved at the low SNR. However, when IMD increases the same $P_{d}$ is achieved at the high SNR. 
The baseband output signal from the LNA considering only the IMD term and the intranetwork interference under two hypotheses is then obtained by using (6) and (24) and is expressed as

$$
y[n]= \begin{cases}w_{a}[n]+\beta_{1} \sum_{k=1}^{K} c_{k}[n]+\frac{3 \beta_{3}}{2} \sum_{k=1}^{K-1} c_{k}^{*}[n] c_{k+1}^{2}[n], & H_{0} \\ w_{a}[n]+\beta_{1}\left(s[n]+\sum_{k=1}^{K} c_{k}[n]\right)+\frac{3 \beta_{3}}{2} \sum_{k=1}^{K-1} c_{k}^{*}[n] c_{k+1}^{2}[n], & H_{1}\end{cases}
$$

The received signal at a particular SOI for the first case under two hypotheses is given by

$$
\begin{aligned}
& y_{\mathrm{SOI}}[n] \\
& = \begin{cases}w_{a}[n]+\left(\beta_{1} c_{z}[n]+\frac{3 \beta_{3}}{2} c_{x}^{*}[n] c_{y}^{2}[n]\right), & H_{0}, \\
w_{a}[n]+\left(\beta_{1}\left(s[n]+c_{z}[n]\right)+\frac{3 \beta_{3}}{2} c_{x}^{*}[n] c_{y}^{2}[n]\right), & H_{1},\end{cases}
\end{aligned}
$$

where $c_{x}$ and $c_{y}$ are the interferers whose third-order IMD falls in the SOI, $c_{z}$ is the interfering signal at SOI, $x, y, z \in K$, and $x \neq y \neq z$.

CR Transmission on the Same Frequency Band. We assume that $N$ number of channels out of $M$ are being allocated by $K$ number of users and are uniformly used by $K$ CRs. Therefore, in each channel, there are $\lfloor K / N\rfloor$ number of CRs, where $\lfloor\cdot\rfloor$ denotes the floor operation. Then, the received complex baseband equivalent wideband signal under two hypotheses is written as

$$
y[n]= \begin{cases}w_{b}[n]+\sum_{i=1}^{N} \sum_{j=1}^{\lfloor K / N\rfloor} c_{i j}[n], & H_{0}, \\ w_{b}[n]+s[n]+\sum_{i=1}^{N} \sum_{j=1}^{\lfloor K / N\rfloor} c_{i j}[n], & H_{1},\end{cases}
$$

where $c_{i j}$ is the interfering signal where $i$ and $j$ are just the index variable, $i \in M, j \in K$.

When $M=N$, we have a similar case to the previous one in which each CR transmits in separate band. Now if we focus on a particular SOI after proper digital filtering, sampling at rate $1 / T_{s}$, and downconversion to intermediate frequency $f_{\mathrm{IF}}$, we further investigate the three following cases:

(i) Noise only case.

(ii) Noise with IMD.

(iii) Noise with IMD plus interferer.

(iv) Noise with interferer.

The first case represents basic hypothesis problem; the fourth case has been already analyzed in the literature. Our focus is on the second and third case. The second case is previously discussed in Section 3.3. For the third case, the signal at the output of the LNA is given by

$$
y_{\mathrm{SOI}}[n]= \begin{cases}w_{a}[n]+\left(\beta_{1} \sum_{j=1}^{\lfloor K / N\rfloor} c_{z j}[n]+\frac{3 \beta_{3}}{2} \sum_{j=1}^{\lfloor K / N\rfloor} c_{x j}^{*}[n] c_{y j}^{2}[n]\right), & H_{0}, \\ w_{a}[n]+\left(\beta_{1}\left(s[n]+\sum_{j=1}^{\lfloor K / N\rfloor} c_{z j}[n]\right)+\frac{3 \beta_{3}}{2} \sum_{j=1}^{\lfloor K / N\rfloor} c_{x j}^{*}[n] c_{y j}^{2}[n]\right), & H_{1},\end{cases}
$$

where $c_{x j}$ and $c_{y j}$ are the interfering signal whose third-order IMD term falls in the SOI; $c_{z j}$ are the interfering signal in the SOI.

$P_{d}$ and $P_{f a}$ under LNA Nonlinearity in Poisson Field of Interferer. It is assumed that the signal, the IMD term, the noise, and the interference are all mutually statistically independent. We model the interference as the Gaussian distribution. Using the CLT in the test statistics, we approximate the test statistics under two hypotheses as

$$
\begin{aligned}
& f_{R \mid H_{0}}(r) \\
& \quad \sim \mathcal{N}\left(\sigma_{n}^{2}+\sigma_{\text {imd }}^{2}+\sigma_{i}^{2}, \frac{2\left(\sigma_{n}^{2}+\sigma_{\text {imd }}^{2}+\sigma_{i}^{2}\right)^{2}}{N}\right),
\end{aligned}
$$

where $\sigma_{i}^{2}$ is the interference variance. 
And

$$
f_{R \mid H_{1}}(r) \sim \mathcal{N}\left(\sigma_{y}^{2}, \frac{2 \sigma_{y}^{4}}{N}\right),
$$

where $\sigma_{y}^{2}=\sigma_{s}^{2}+\sigma_{n}^{2}+\sigma_{\text {imd }}^{2}+\sigma_{i}^{2}$.

After some derivation, we can derive $P_{d}$ and $P_{\mathrm{fa}}$ as

$$
P_{d}=Q\left(\sqrt{\frac{N}{2}}\left(\frac{\lambda_{\mathrm{ED}}}{\left(\sigma_{n}^{2}+\sigma_{s}^{2}+\sigma_{\mathrm{imd}}^{2}+\sigma_{i}^{2}\right)}-1\right)\right),
$$

where the signal to interference ratio (SIR) is defined as $\sigma_{s}^{2} / \sigma_{i}^{2}$

$$
P_{\mathrm{fa}}=Q\left(\frac{\lambda_{\mathrm{ED}}-\left(\sigma_{n}^{2}+\sigma_{\mathrm{imd}}^{2}+\sigma_{i}^{2}\right)}{\left(\sigma_{n}^{2}+\sigma_{\mathrm{imd}}^{2}+\sigma_{i}^{2}\right) / \sqrt{N / 2}}\right) .
$$

Under $H_{0}$ hypothesis, for a fixed $P_{\mathrm{fa}}$, the threshold is derived as

$$
\lambda_{\mathrm{ED}}=\left(\sigma_{n}^{2}+\sigma_{\mathrm{imd}}^{2}+\sigma_{i}^{2}\right)\left(1+\frac{Q^{-1}\left(P_{\mathrm{fa}}\right)}{\sqrt{N / 2}}\right) .
$$

$\lambda_{\mathrm{ED}}$ in (33) indicates that the threshold estimation is dependent on the SNR, the number of the samples, the required false alarm probability, the IMD variance, and the interference variance.

3.6. Simulation Results. The interfering CRs are generated randomly according to the Poisson distribution with density $\lambda_{\text {IN }}=100,000$ persons $/ \mathrm{km}^{2}$. The distance between PU and $\mathrm{CR}_{0} d_{\mathrm{PU}}$ can be varied. We show result at the distance $d_{\mathrm{PU}}=600 \mathrm{~m}$. Later, we show the result at $d_{\mathrm{PU}}=500 \mathrm{~m}$ that shows that the results have the same trend when $d_{\mathrm{PU}}$ varied. Moreover, if $\lambda_{\mathrm{IN}}$ is increased, the effect of interference is more severe and vice versa given that the distance between $\mathrm{PU}$ and $\mathrm{CR}_{0}$ is kept unchanged in both the simulations. The effect of varying number of samples is discussed later. Since the theoretical derivation is carried out without the effect of shadowing, the environment effect has been removed in the simulated plot. Hence, the theoretical and simulated curves are the same. This also provides the validation of the simulated and the theoretical results. The plot obtained is shown in Figure 7.

3.7. Effect of Varying Number of Samples. The simulations in this part are for the importance of the sensing time (interval); that is, it improves the performance of the ED. Sensing time plays an important role in improving the performance of the ED. We plot the effect of the observation interval $(N)$ on $P_{d}$. $P_{d}$ depends upon $N$. This is due to the fact that increasing $N$ results in the increase in the effective SNR or SIMDR $[15,20]$. It is noted that the receiver operating under the high input power experiences more severe nonlinearity behavior. Therefore, the plots obtained in Figures 8, 9, and 10 are further extended negatively, implying a large sensing time, to get the desired $P_{d}$. The plot in Figure 8 is plotted with parameters $\mathrm{SNR}=3 \mathrm{~dB}, \mathrm{SBR}=-23.98 \mathrm{~dB}, \mathrm{SIR}=-16.47 \mathrm{~dB}$,

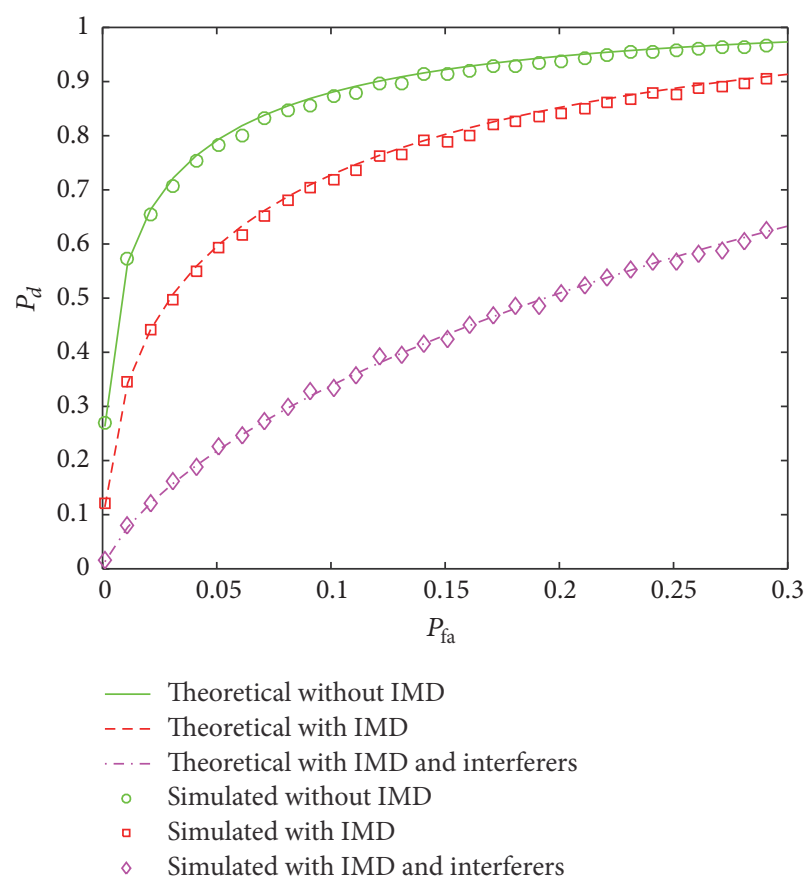

Figure 7: $P_{d}$ versus $P_{\mathrm{fa}}$.

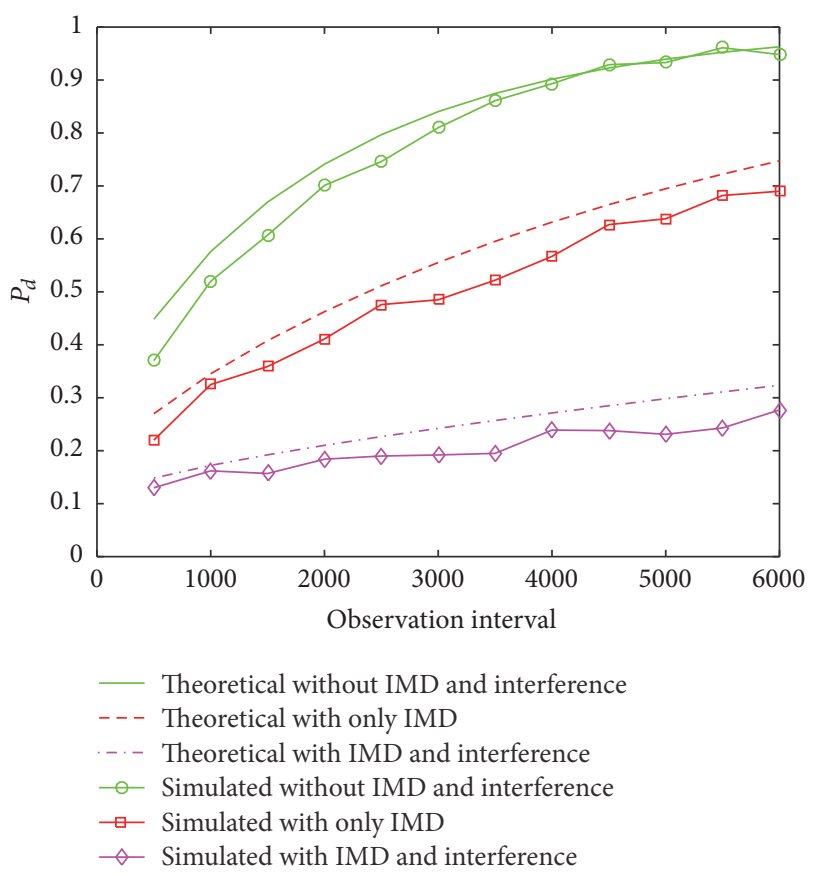

FIGURE 8: Effect of sensing time on the performance of ED at $P_{\mathrm{fa}}=$ 0.1 .

SIMD $=-14.86 \mathrm{~dB}, P_{\mathrm{fa}}=0.1$, and PU at $600 \mathrm{~m}$. In order to gain insight into the theory, the following simulations are done for a variety of different cases. Keeping all the other parameters unchanged, we adjust the value of $P_{\mathrm{fa}}$ to visualize its effect on the performance. Figure 9 is plotted for $P_{\mathrm{fa}}=$ 0.05 . The result clearly indicates the fact that when $P_{\mathrm{fa}}$ is decreased, the overall curve shifts towards low $P_{d}$ at low 


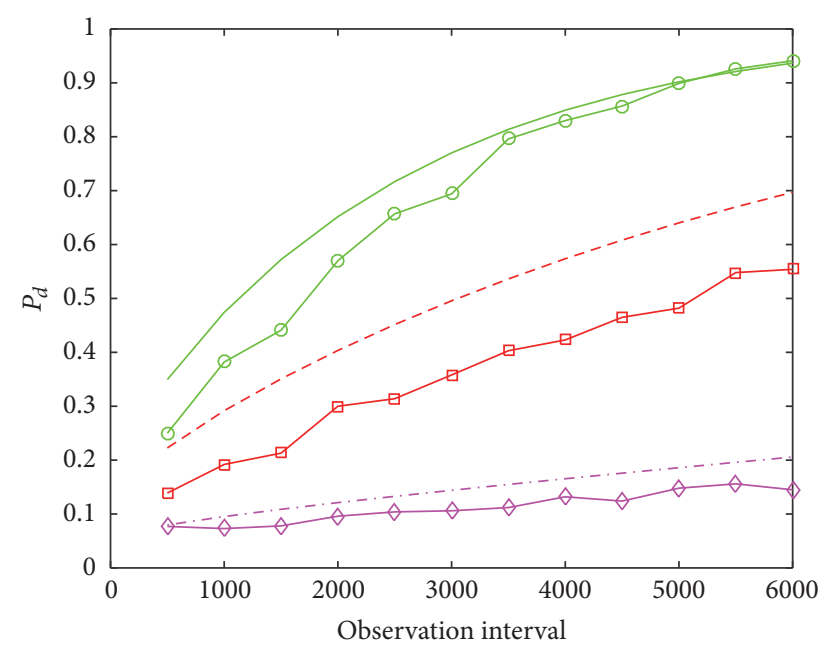

- Theoretical without IMD and interference
-- - Theoretical with only IMD
-- - Theoretical with IMD and interference
- Simulated without IMD and interference
$\square-$ Simulated with only IMD
$\neg-$ Simulated with IMD and interference

Figure 9: Effect of sensing time on the performance of ED at $P_{\mathrm{fa}}=$ 0.05 .

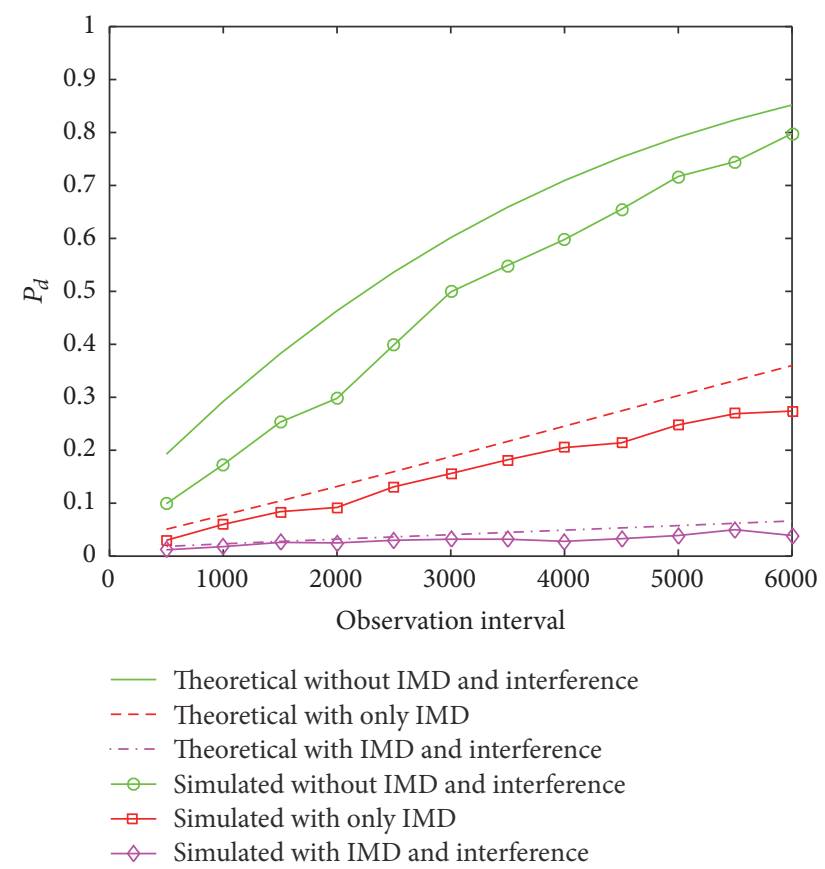

Figure 10: Effect of sensing time on the performance of ED at $P_{\mathrm{fa}}=$ 0.01 .

observation interval. Figure 10 is plotted for $P_{\mathrm{fa}}=0.01$. It is seen that the overall curve shifts towards much lower $P_{d}$ at lower observation interval.

The theoretical curves and the simulated ROC curves in Figures 8, 9, and 10 are somewhat different from the simulated curves because the simulated results are plotted considering

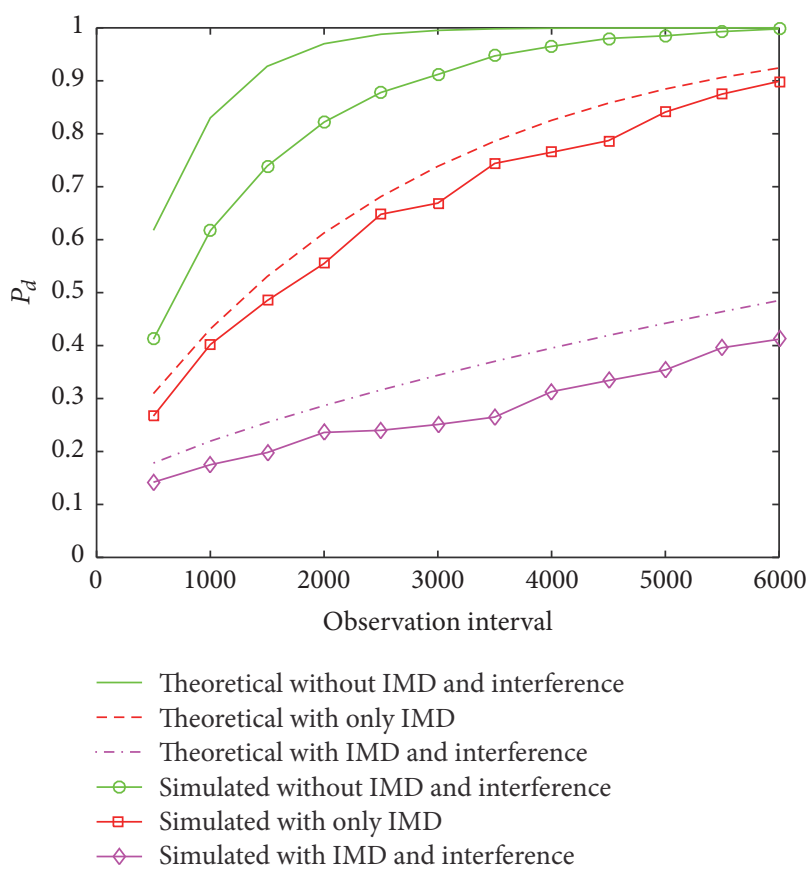

Figure 11: Observation interval versus $P_{d}$ with $\mathrm{PU}$ at $500 \mathrm{~m}$.

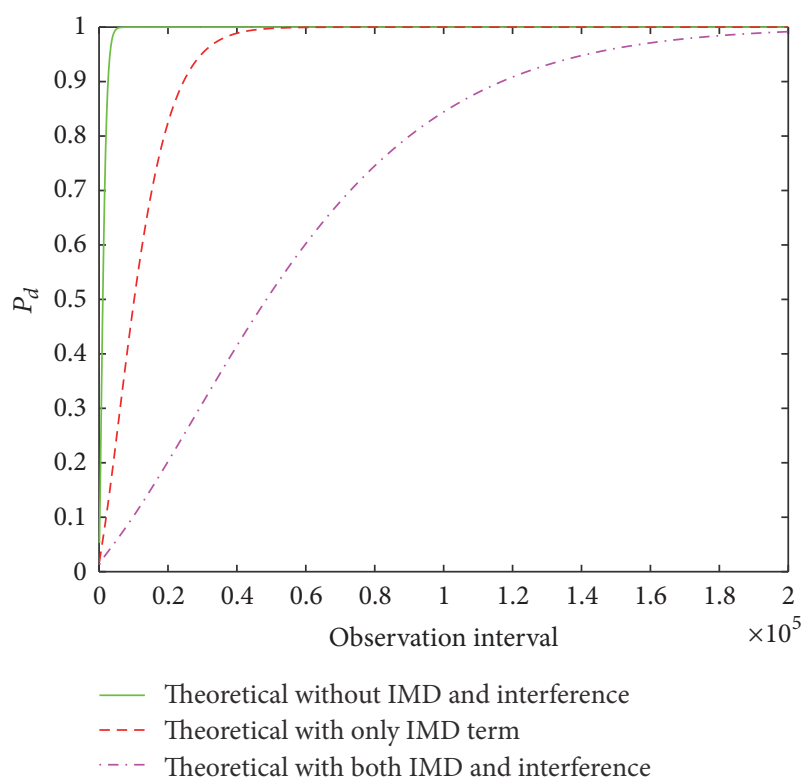

Figure 12: Observation interval versus $P_{d}$ at $P_{\mathrm{fa}}=0.01$.

the effect of environment, that is, shadowing. Hence, the theoretical results can be considered as the upper bound of the performance. Figure 11 shows the situation when the PU distance is decreased to $500 \mathrm{~m}$ while keeping all the other parameters unchanged for $P_{\mathrm{fa}}=0.1$. In order to get the estimation of the total observation time interval required to achieve the required $P_{d}$, we plot the theoretical curves to visualize the upper bound on $N$. Figure 12 is plotted for $P_{\mathrm{fa}}=0.01$ to determine the required $P_{d}$ in different scenarios. Figure 12 clearly shows that the observation interval in the 


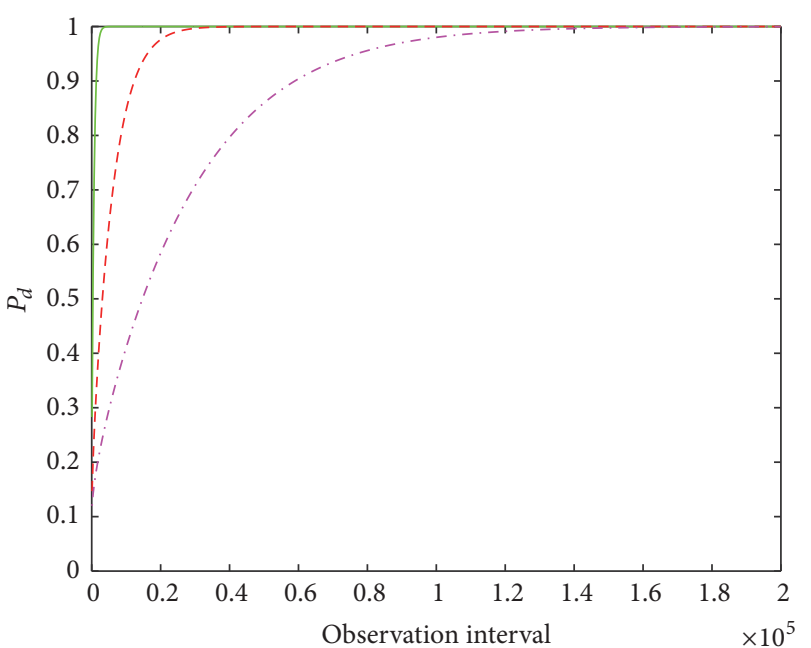

Theoretical without IMD and interference
- - Theoretical with only IMD term
-.. - Theoretical with both IMD and interference

Figure 13: Observation interval versus $P_{d}$ at $P_{\mathrm{fa}}=0.1$.

case of the interference from the secondary network considered together with the IMD term is much larger than the one in the case with the IMD term only. This large observation interval required for detection in the case of interference from secondary network may lead to impractical CR. Intuitively, if the requirement of the false alarm probability is increased to $P_{\mathrm{fa}}=0.1$, the sensing time is reduced for a particular value of the detection probability as shown in Figure 13.

\subsection{Simulation Result for Threshold Selection with LNA Non-} linearity. The actual distribution of the statistic of threshold is determined by the correlated Gaussian sequence instead of the white Gaussian sequence. This is due to the nonlinearity of LNA acting on the white Gaussian noise in the absence of PU signal. Figure 14 shows the fitting of the simulated Gaussian noise after the LNA with the correlated Gaussian sequence. The simulated plot in Figure 14 shows that the correlated Gaussian probability density function (pdf) fits the pdf of the nonlinear filtered noise better than the uncorrelated Gaussian pdf [18]. If the threshold is selected based only on Gaussian process for all the subbands, then the performance of ED is degraded. However, it is noted that this happens only when we consider the effect of nonlinearity. When we consider the nonlinearity of LNA, the output of the LNA is not just the white noise sequence but the summation of correlated non-Gaussian and Gaussian process which is approximated using multivariate Gaussian mixtures. Figure 15 depicts the threshold statistics considering the output of the LNA with the multivariate Gaussian mixture. It is clearly seen that the threshold is well approximated by the multivariate Gaussian mixture rather than the Gaussian process which happens to be constant for each frequency band.

As shown in Figure 16, the selected threshold level brings the probability of threshold being exceeded, when only noise is present. The value matches the target $P_{\mathrm{fa}}$ that is set to

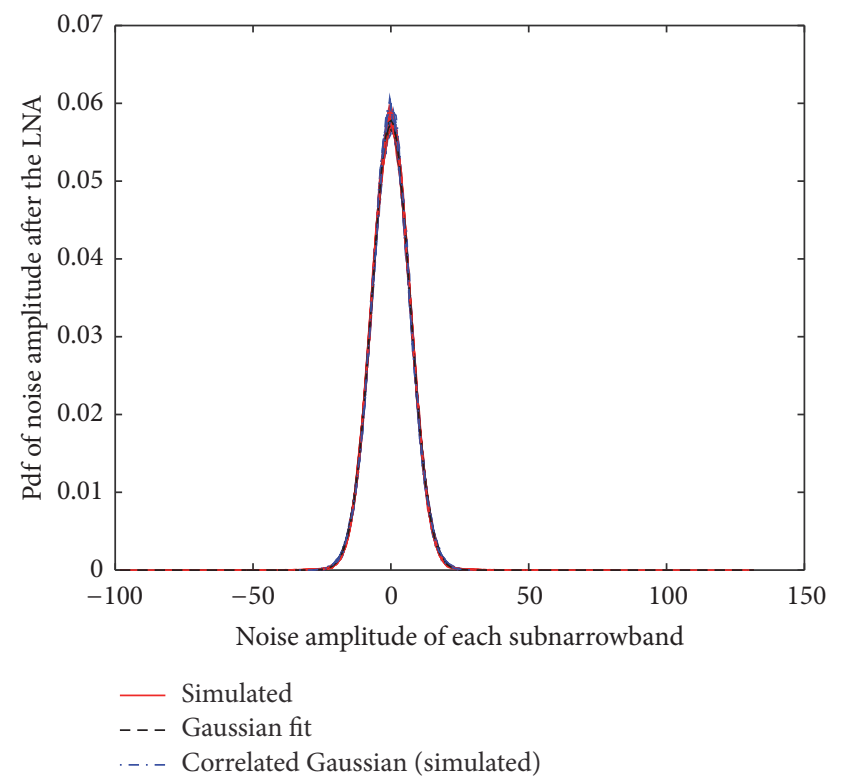

FIgURE 14: Pdf of the noise amplitude after nonlinear LNA.

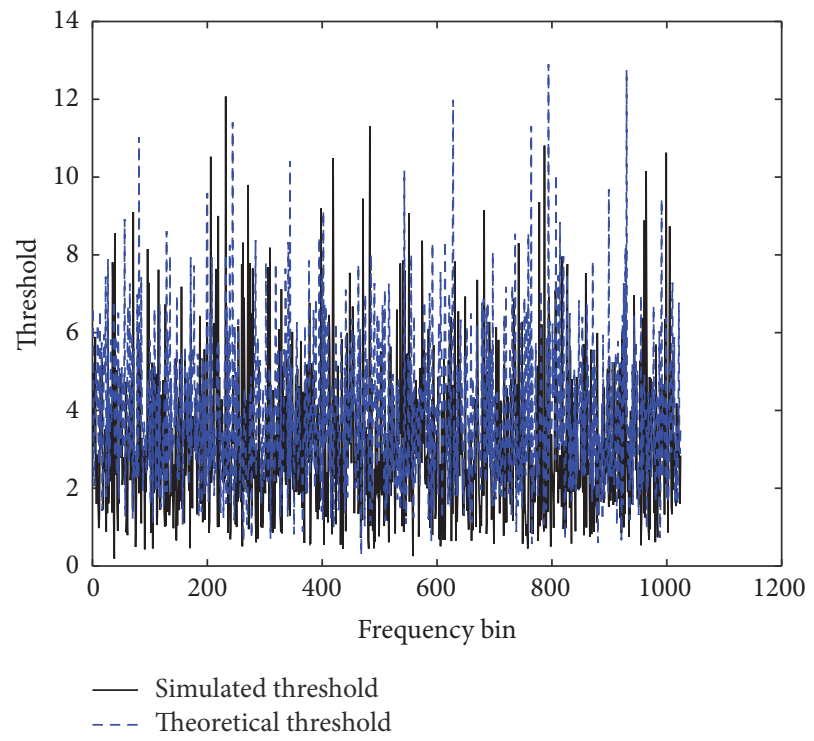

FIGURE 15: Approximation of simulated threshold value with multivariate Gaussian mixture.

0.1. Due to a finite number of samples being used in the simulation, the probability of threshold being exceeded in the presence of only noise cannot exactly be equal to the target $P_{\mathrm{fa}}$. The similar simulation for the case of the target $P_{\mathrm{fa}}$ set to 0.01 is shown in Figure 17.

\section{Conclusion}

The effect of nonlinearity due to the LNA was first considered to visualize its impact on the spectrum sensing. It was seen that the effect of the LNA nonlinearity degrades the performance of ED. It was also examined that the performance of ED depends upon various factors, that is, SNR, the power 


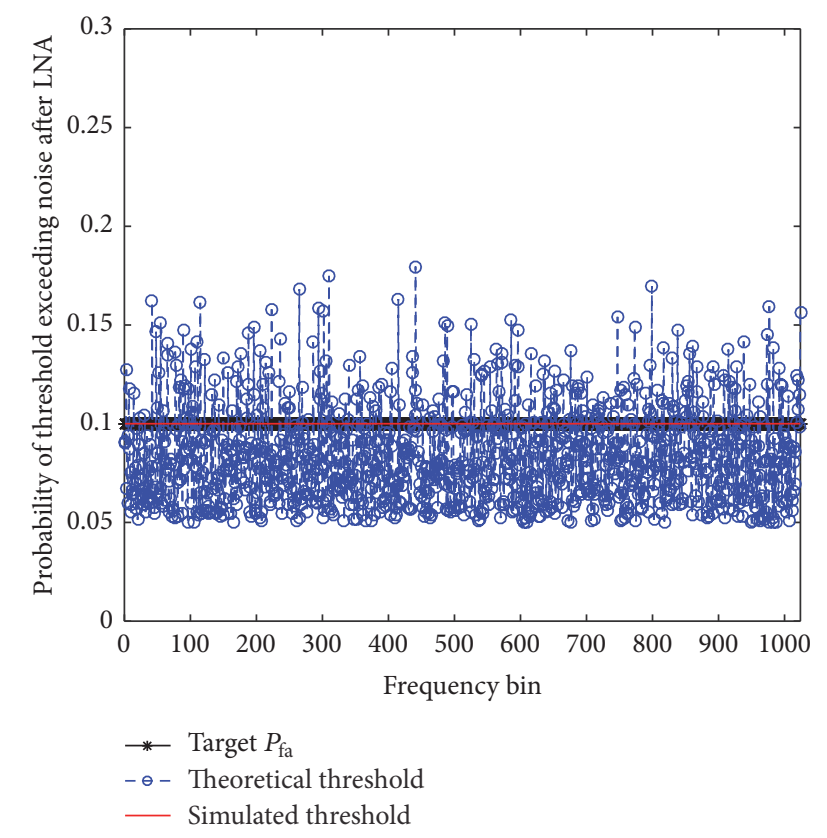

FIgURE 16: Probability of threshold being exceeded only in presence of noise at $P_{\mathrm{fa}}=0.1$.

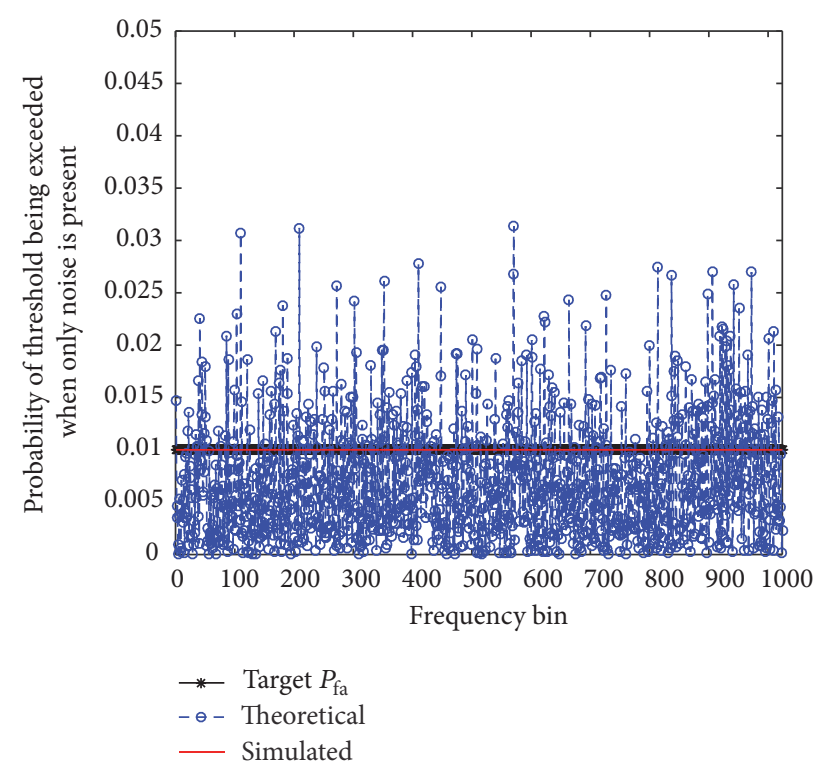

FIgURE 17: Probability of threshold being exceeded only in presence of noise at $P_{\mathrm{fa}}=0.01$.

of IMD, and interfering signal, and the observation interval. In reality, the sensing time in which the $\mathrm{CR}$ is required to sense the channel is the fundamental limit that needs to be considered for providing a good quality of service to the users as well as for practical implementation of CR devices.

The analysis in the wideband spectrum sensing along with the effect of interference generated from the other CRs in the secondary network was then performed. For simplicity, the approximations while performing theoretical analysis were based on the assumption of Gaussian distribution but simulations were carried out with and without the shadowing effect. The effect of shadowing was clearly seen in the simulation results. The main contribution of this paper was to analyze the performance degradation in spectrum sensing due to inherent nonlinearity of an LNA in the Poisson field of interferer considering intranetwork interference, which models the more realistic scenarios than the ones in the existing literatures $[10,15]$. It was also shown that the noise model after LNA is more accurate by using the multivariate Gaussian mixture model. A fixed threshold setting for the entire wideband seems to be inappropriate resulting in performance degradation. Therefore, an adaptive threshold setting based on the multivariate Gaussian mixture model was proposed to increase the overall performance of the CR.

\section{Competing Interests}

The authors declare that they have no competing interests.

\section{Acknowledgments}

The first author would like to thank Asian Development Bank-Japan Scholarship Program (ADB-JSP) for providing him with the grant for pursuing his M.E. degree in Asian Institute of Technology.

\section{References}

[1] M. Marcus, J. Burtle, B. Franca, A. Lahjouji, and N. McNeil, Report of the Unlicensed Devices and Experimental Licenses Working Group, Federal Communications Commission, Spectrum Policy Task Force, 2002.

[2] H. Sun, A. Nallanathan, C.-X. Wang, and Y. Chen, "Wideband spectrum sensing for cognitive radio networks: a survey," IEEE Wireless Communications, vol. 20, no. 2, pp. 74-81, 2013.

[3] A. Sahai and D. Cabric, "Spectrum sensing: fundamental limits and practical challenges," in Proceedings of the IEEE International Symposium on New Frontiers in Dynamic Spectrum Access Networks (DySPAN '05), 2005.

[4] B. Razavi, "Design considerations for direct-conversion receivers," IEEE Transactions on Circuits and Systems II: Analog and Digital Signal Processing, vol. 44, no. 6, pp. 428-435, 1997.

[5] M. Allen, J. Marttila, M. Valkama, S. Makinen, M. Kosunen, and J. Ryynanen, "Digital linearization of direct-conversion spectrum sensing receiver," in Proceedings of the 1st IEEE Global Conference on Signal and Information Processing (GlobalSIP '13), pp. 1158-1161, Austin, Tex, USA, December 2013.

[6] Z. Chen, C.-X. Wang, X. Hong, J. Thompson, S. A. Vorobyov, and X. Ge, "Interference modeling for cognitive radio networks with power or contention control," in Proceedings of the IEEE Wireless Communications and Networking Conference (WCNC '10), Sydney, Australia, April 2010.

[7] M. Derakhshani, T. Le-Ngoc, and M. Vu, "Interference and outage analysis in a cognitive radio network with beacon," in Proceedings of the 25th Biennial Symposium on Communications (QBSC '10), pp. 261-264, IEEE, Ontario, Canada, May 2010.

[8] N. Mayekar and A. M. Wyglinski, "Interference performance evaluation of secondary users in cognitive radio networks," in 
Proceedings of the IEEE 81st Vehicular Technology Conference (VTC Spring '15), 2015.

[9] Q. Liu, Z. Zhou, C. Yang, and Y. Ye, "Outage probability analysis for cognitive radio network caused by primary users," in Proceedings of the 3rd IEEE International Conference on Communications and Networking in China (ChinaCom '08), pp. 1323-1326, Hangzhou, China, August 2008.

[10] A. U. Makarfi and K. A. Hamdi, "Efficiency of energy detection for spectrum sensing in a Poisson field of interferers," in Proceedings of the IEEE Wireless Communications and Networking Conference (WCNC '12), pp. 1023-1028, April 2012.

[11] M. Valkama, M. Renfors, and V. Koivunen, "Advanced methods for I/Q imbalance compensation in communication receivers," IEEE Transactions on Signal Processing, vol. 49, no. 10, pp. 23352344, 2001.

[12] J. Verlant-Chenet, J. Renard, J.-M. Dricot, P. De Doncker, and F. Horlin, "Sensitivity of spectrum sensing techniques to RF impairments," in Proceedings of the IEEE 71st Vehicular Technology Conference (VTC-Spring '10), Taipei, Taiwan, May 2010.

[13] V. Syrjälä, M. Valkama, N. N. Tchamov, and J. Rinne, "Phase noise modelling and mitigation techniques in OFDM communications systems," in Proceedings of the 2009 Wireless Telecommunications Symposium (WTS '09), Prague, Czech Republic, April 2009.

[14] E. Rebeiz, A. S. H. Ghadam, M. Valkama, and D. Cabric, "Suppressing RF front-end nonlinearities in wideband spectrum sensing," in Proceedings of the 8th International Conference on Cognitive Radio Oriented Wireless Networks and Communications (CROWNCOM '13), pp. 87-92, July 2013.

[15] E. Rebeiz, A. S. Hagh Ghadam, M. Valkama, and D. Cabric, "Spectrum sensing under RF non-linearities: performance analysis and DSP-enhanced receivers," IEEE Transactions on Signal Processing, vol. 63, no. 8, pp. 1950-1964, 2015.

[16] B. Razavi, RF Microelectronics, University of California, Los Angeles, Calif, USA; Pearson Education, Upper Saddle River, NJ, USA, 2012.

[17] R. Tandra and A. Sahai, "SNR walls for signal detection," IEEE Journal on Selected Topics in Signal Processing, vol. 2, no. 1, pp. 4-17, 2008.

[18] A. Taparugssanagorn, K. Umebayashi, J. Lehtomaki, and C. Pomalaza-Raez, "Analysis of the effect of nonlinear low noise amplifier with memory for wideband spectrum sensing," in Proceedings of the 1st International Conference on $5 \mathrm{G}$ for Ubiquitous Connectivity (5GU '14), pp. 87-91, Levi, Finland, November 2014.

[19] A. Gorcin, K. A. Qaraqe, H. Celebi, and H. Arslan, "An adaptive threshold method for spectrum sensing in multi-channel cognitive radio networks," in Proceedings of the 17th International Conference on Telecommunications (ICT '10), pp. 425-429, April 2010.

[20] K. Hamdi and K. B. Letaief, "Power, sensing time, and throughput tradeoffs in cognitive radio systems: a cross-layer approach," in Proceedings of the IEEE Wireless Communications and Networking Conference (WCNC '09), Budapest, Hungary, April 2009. 


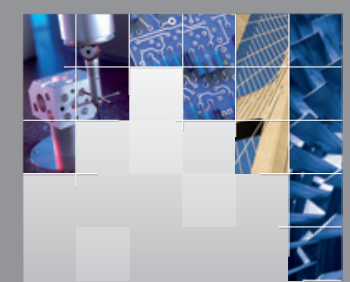

\section{Enfincering}
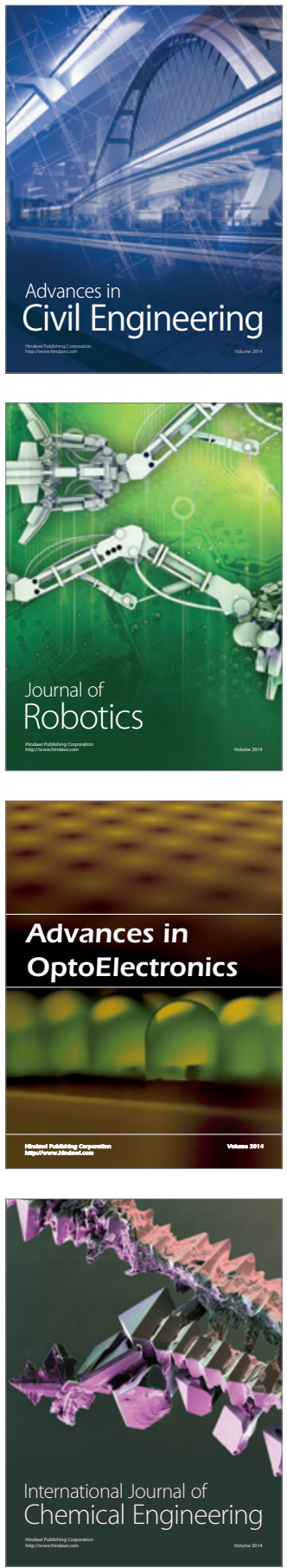

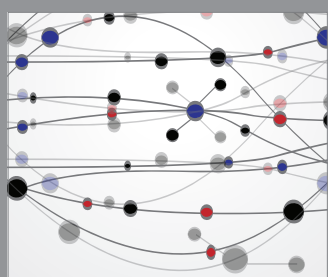

The Scientific World Journal

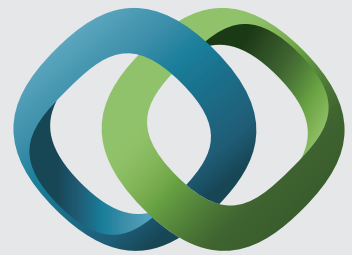

\section{Hindawi}

Submit your manuscripts at

http://www.hindawi.com
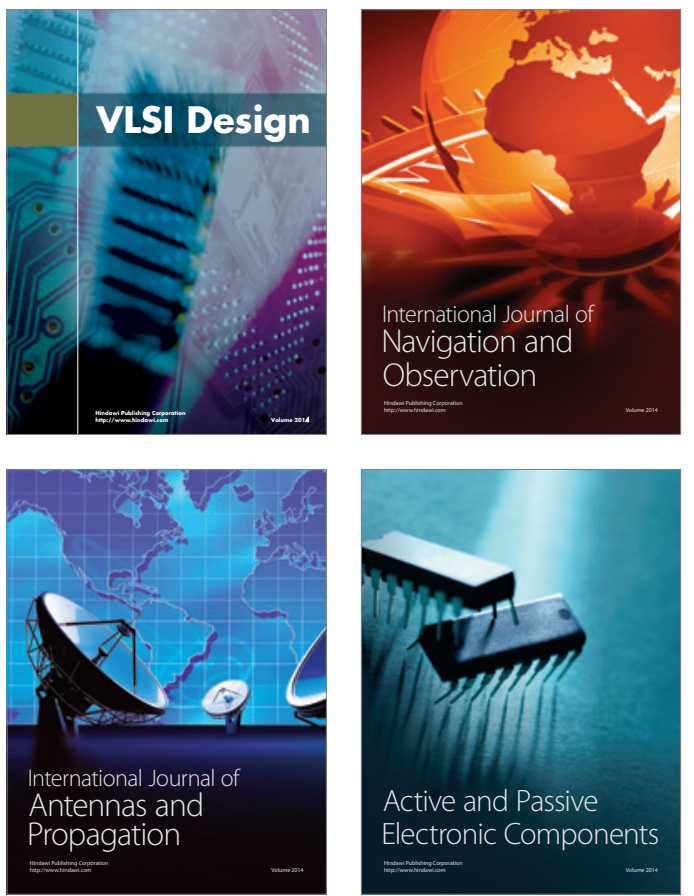
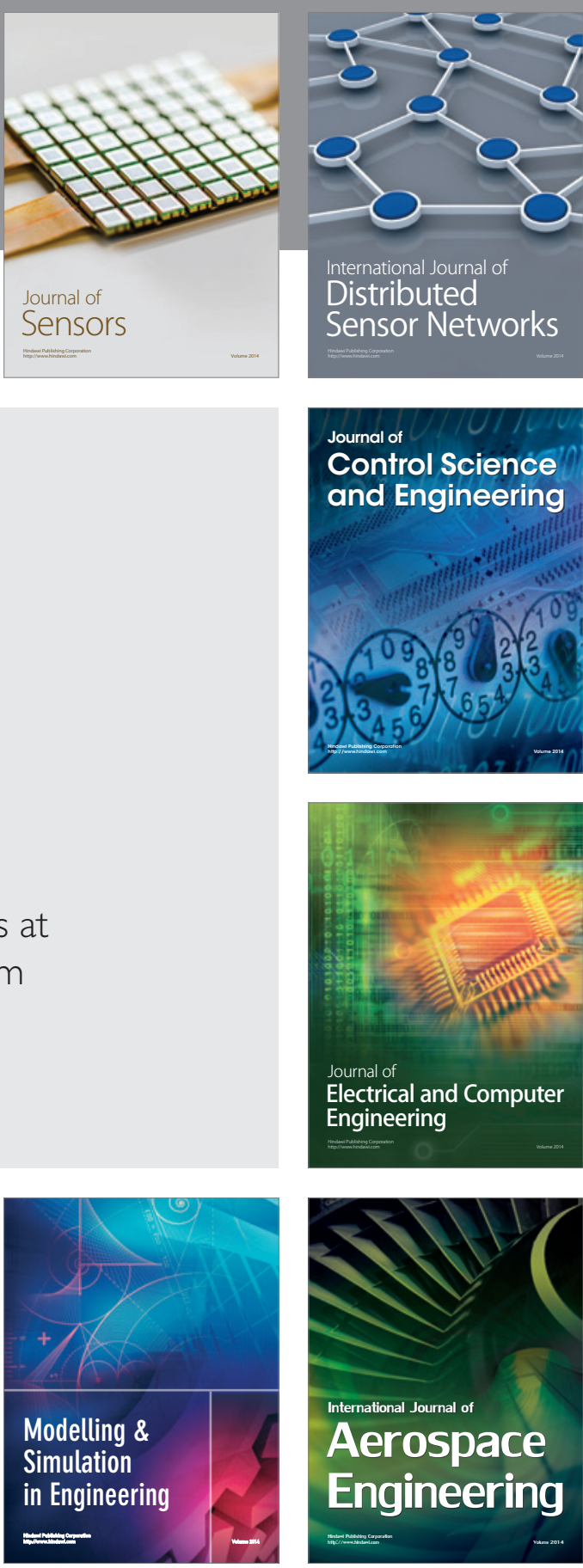

International Journal of

Distributed

Sensor Networks

Journal of

Control Science

and Engineering
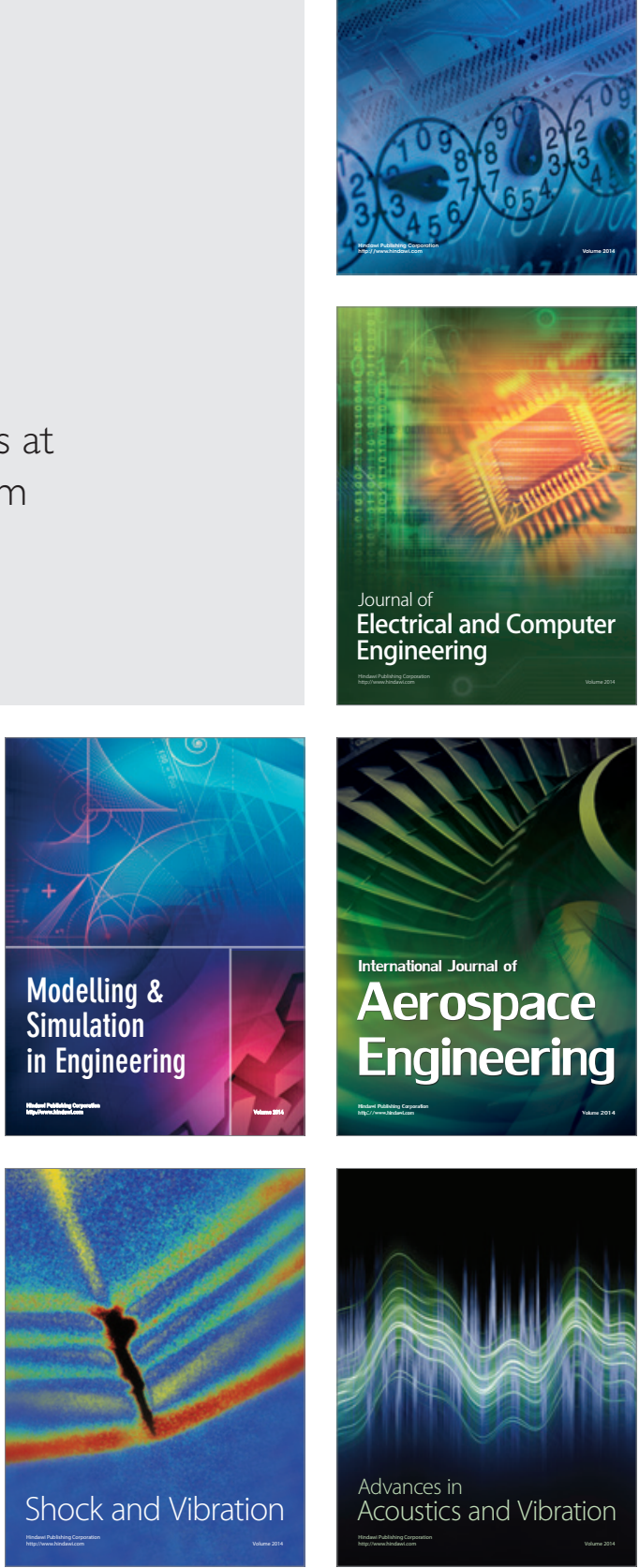